\title{
Salvage therapy with mitoxantrone, etoposide, bleomycin and dexamethasone for refractory or relapsed aggressive non-Hodgkin's lymphoma patients with a poor performance status or comorbidity
}

\author{
XUEDE LIN*, XI SHI* ${ }^{*}$ WUCHA ZENG, MIN ZHENG and LIMING HUANG \\ Department of Chemotherapy, The First Affiliated Hospital, Fujian Medical University, Fuzhou, Fujian 350005, P.R. China
}

Received January 3,2014; Accepted August 7, 2014

DOI: $10.3892 / \mathrm{ol} .2014 .2517$

\begin{abstract}
The treatment of refractory or relapsed aggressive nonHodgkin's lymphoma (NHL) in patients in a state of poor health is difficult due to their ineligibility to receive intensive salvage chemotherapy. In the present study, 16 refractory or relapsed aggressive NHL patients with a poor performance status or comorbidities were treated with mitoxantrone, etoposide, bleomycin and dexamethasone (MEBD) therapy. The treatment consisted of $10 \mathrm{mg} / \mathrm{m}^{2}$ intravenous (IV) mitoxantrone on day $1,75 \mathrm{mg} / \mathrm{m}^{2}$ IV etoposide on days 1-3, $20 \mathrm{mg}$ IV dexamethasone on days 1-4 and $15 \mathrm{mg}$ intramuscular bleomycin on days $1,4,8$ and 12 , every 21 days. The efficacy and toxicity of the regimen were evaluated. The overall response rate was $68.8 \%$, with a complete response rate of $18.8 \%$ and a partial response rate of $50.0 \%$. The efficacy of the treatment for B-cell lymphoma was greater than that for T-cell lymphoma. The median progression-free survival time for the patients was 16.7 months and the median overall survival time was 22.4 months. The one-year overall survival rate was $62.5 \%$ and the two-year overall survival rate was $43.8 \%$. The most common toxicity symptom was myelosuppression. In conclusion, refractory or relapsed aggressive NHL patients with a poor performance status or comorbidity are eligible for chemotherapy. MEBD therapy is an effective and feasible salvage regimen for NHL patients in a state of poor health.
\end{abstract}

\section{Introduction}

The majority of aggressive non-Hodgkin's lymphoma (NHL) cases originate from $\mathrm{B}$ cells, with $\sim 10 \%$ arising from T cells (1).

Correspondence to: Dr Xi Shi, Department of Chemotherapy, The First Affiliated Hospital, Fujian Medical University, 20 Chazhong Road, Fuzhou, Fujian 350005, P.R. China

E-mail: xishi9999@163.com

*Contributed equally

Key words: mitoxantrone, salvage therapy, non-Hodgkin's lymphoma, poor performance status, comorbidity
The standard first-line chemotherapy for the majority of aggressive NHL cases is cyclophosphamide, doxorubicin, vincristine and prednisolone (CHOP) or R-CHOP, a combination of $\mathrm{CHOP}$ and rituximab, a monoclonal antibody to cluster of differentiation $20(2,3)$. Although the majority of patients with aggressive NHL are responsive to the initial chemotherapy, 40 to $60 \%$ either fail to achieve a complete response (CR) following firstline chemotherapy or relapse subsequent to obtaining CR (4).

The current standard treatment strategy for refractory or relapsed NHL is high-dose therapy and autologous stem cell transplantation (HD-ASCT) with curative intent in patients without comorbidities $(5,6)$. However, HD-ASCT is only suitable for fit, young patients who are chemosensitive to salvage chemotherapy. In the absence of hematopoietic stem cell transplantation, the majority of the current treatment strategies for refractory or relapsed NHL are palliative (7-9). The majority of patients are not eligible for ASCT due to refractory disease, age, a poor performance status, comorbidities and other individual reasons $(5,10,11)$. Therefore, alternative salvage approaches have to be employed in these patients. The standard salvage chemotherapy for these NHL patients has not been determined. Prior to the advent of novel chemotherapeutic or targeted agents, the ideal approach for these patients remains as a chemotherapeutic regimen with a high response rate and less toxicity, and containing chemotherapeutic agents that are not cross-resistant to previous therapy.

For refractory or relapsed aggressive NHL patients with a poor performance status or comorbidities, treatment efficacy and quality of life require careful simultaneous consideration. In the present study, the mitoxantrone, etoposide, bleomycin and dexamethasone (MEBD) regimen, which is composed of myelosuppressive (mitoxantrone and etoposide) and non-myelosuppressive (bleomycin and dexamethasone) drugs, was used to treat a group of such patients, and the response rates and toxicities were investigated.

\section{Patients and methods}

Patients. A retrospective analysis of 16 patients treated in the First Affiliated Hospital, Fujian Medical University (Fuzhou, China) between 2009 and 2012 was conducted. All patients had pathologically confirmed aggressive NHL and 
had been previously treated with at least one anthracyclinebased chemotherapeutic agent. All patients had either an Eastern Cooperative Oncology Group (ECOG) performance status (12) of 2.0-4.0 or comorbidities. Among the patients with comorbidities, one presented with bronchiectasis, one with deep venous thrombosis, two with diabetes and five with chronic hepatitis B infection. Patients with primary central nervous system lymphoma or testicular involvement were not included in the present study. Prior to MEBD chemotherapy, all patients were staged according to the Ann Arbor classification (13), with physical examination, bone marrow biopsy and computed tomography (CT) scans of the neck, chest, abdomen and pelvis. Serum lactate dehydrogenase (LDH) and $\beta 2$-microglobulin levels were also analyzed. In addition, baseline electrocardiogram (ECG) and ultrasonic cardiogram examinations were performed. The patients were required to have adequate bone marrow, hepatic and renal function, defined as a white blood cell count of $\geq 3,500 / \mathrm{mm}^{3}$, an absolute neutrophil count of $\geq 1,500 / \mathrm{mm}^{3}$, a platelet count of $\geq 100,000 /$ $\mathrm{mm}^{3}$, alanine aminotransferase or aspartate aminotransferase levels $<2.0$ times the upper normal limit, a bilirubin level of $\leq 1.5$ times the upper normal limit and a serum creatinine level of $\leq 1.5$ times the upper normal limit. This study was approved by the ethics committee of the First Affiliated Hospital, Fujian Medical University.

Treatment schedule. Once written informed consent had been obtained, all patients received systemic chemotherapy with the MEBD regimen, consisting of $10 \mathrm{mg} / \mathrm{m}^{2}$ intravenous (IV) mitoxantrone on day $1,75 \mathrm{mg} / \mathrm{m}^{2} \mathrm{IV}$ etoposide on days $1-3,20 \mathrm{mg}$ IV dexamethasone on days 1-4 and intramuscular $15 \mathrm{mg}$ bleomycin on days $1,4,8$ and 12 , and the cycles were repeated every 21 days. If toxicity occurred, the dose was adjusted according to the physician. If hematological toxicity occurred, prophylactic granulocyte colony-stimulating factor (G-CSF) was used in subsequent cycles. The treatment was continued until either a maximum of six cycles, disease progression, the occurrence of unacceptable toxicity or the decision of the patient to withdraw. During the chemotherapy period, low molecular weight heparin calcium injections $(0.3 \mathrm{ml} ; 3075$ AXaIU, twice daily) were provided for the patient with deep venous thrombosis, insulin was administered to the diabetes patients, with monitored blood glucose levels, and entecavir tablets ( $0.5 \mathrm{mg}$, daily) were used in the patients with hepatitis B infection for 6 months following chemotherapy, with monitored blood hepatitis B virus DNA concentrations.

Response and toxicity evaluation. For the response evaluation, CT scans were performed every two cycles of chemotherapy until the end of the treatment and every two months during follow-up. Bone marrow biopsies were performed every two cycles of chemotherapy until the end of the treatment and according to the physician during follow-up. Blood, ECG and ultrasonic cardiogram results were monitored for signs of hematological or cardiac toxicity. Tumor responses, including complete response (CR), partial response (PR), stable disease (SD) and progressive disease (PD) were assessed by the Response Evaluation Criteria In Solid Tumors (14). The overall response rate (ORR) was calculated as the CR plus PR. The progression-free survival (PFS) time was calculated as the time period between the date of MEBD chemotherapy and the date of disease progression. The overall survival (OS) time was calculated as the time period between the initial MEBD treatment and the time-point at which the patient succumbed to the disease. Toxicity was graded by the National Cancer Institute Common Terminology Criteria, version 3.0 (15).

\section{Results}

Patient characteristics. A total of 16 refractory or relapsed aggressive NHL patients with a poor performance status or comorbidity were treated. The baseline characteristics of the patients and the previous treatments received are summarized in Table I. The median age of the patients was 55 years (range, 35-79 years), and 11 patients were male and five were female. Seven patients had ECOG performance status scores of 2.0-4.0. Nine patients presented with comorbidities. Histologically, 11 patients $(68.8 \%)$ presented with diffuse large B-cell lymphoma (DLBCL) and five patients (31.2\%) with peripheral T-cell lymphoma (PTCL). Two patients $(12.5 \%)$ were at stage I or II and 14 patients $(87.5 \%)$ were at stage III or IV. A total of 13 patients $(81.3 \%)$ had elevated serum LDH levels and all 16 patients $(100 \%)$ had elevated serum $\beta 2$-microglobulin levels. With regard to previous chemotherapy, 13 patients (81.3\%) had been administered CHOP chemotherapy, two patients $(12.5 \%)$ had received R-CHOP chemotherapy and one patient $(6.25 \%)$ had experienced CHOP + etoposide chemotherapy. Among these patients, six (37.5\%) had been treated by at least one further regimen in addition to anthracycline-based chemotherapy. Six patients $(37.5 \%)$ were refractory to previous chemotherapy and 10 patients $(62.5 \%)$ had relapsed subsequent to previous chemotherapy.

Treatment response and survival time. Out of the 16 patients, three $(18.8 \%)$ achieved a CR, eight $(50.0 \%)$ obtained PR, one $(6.2 \%)$ exhibited SD and four $(25.0 \%)$ developed PD. The ORR (CR + PR) was $68.8 \%$. Among the 11 DLBCL patients, a CR was achieved in $18.2 \%(2 / 11)$ and PR in $63.6 \%(7 / 11)$; thus ORR was reached in $81.8 \%$ patients $(9 / 11)$. Among the five PTCL patients, the CR rate was $20.0 \%$ (1/5), the PR rate was $20.0 \%(1 / 5)$ and the ORR was therefore $40.0 \%$. The patients with refractory and relapsed aggressive NHL were all responsive to the MEBD chemotherapy. The treatment results are summarized in Table II. The median PFS time was 16.7 months and the median OS time was 22.4 months. The one-year overall survival rate was $62.5 \%$ and the two-year overall survival rate was $43.8 \%$. At present, 10 patients remain alive and three of these patients remain with a CR. One patient has remained in CR for 47 months thus far.

Toxicity. The side-effects of chemotherapy are presented in Table III. The hematological toxicity was severe, with grade $3 / 4$ neutropenia observed in 11 patients $(68.8 \%)$ and two febrile neutropenia cases $(12.5 \%)$. Grade 3/4 thrombocytopenia occurred in $18.8 \%$ of cases, but only grade $1 / 2$ anemia was observed. The majority of non-hematological toxicity consisted of hepatic dysfunction and gastrointestinal reactions, which were mild and transient. Grade 1/2 interstitial pneumonia occurred in four patients $(25.0 \%)$. Grade 1 arrhythmia 
Table I. Patient characteristics.

\begin{tabular}{lc}
\hline Characteristic & Value \\
\hline Median age (range), years & $55(35-79)$ \\
Gender, $\mathrm{n}$ & \\
Male & 11 \\
Female & 5 \\
ECOG performance status, $\mathrm{n}$ & \\
0-1 & 9 \\
2-4 & 7 \\
Stage, $\mathrm{n}$ & \\
I/II & 2 \\
III/IV & 14 \\
B-symptoms, & 9 \\
LDH level & \\
Elevated & 13 \\
Normal & 3
\end{tabular}

$\beta 2$-microglobulin level, $\mathrm{n}$

Elevated

Normal

16

Bulky mass, $\mathrm{n}$

Bone marrow involvement, $\mathrm{n}$

Disease status, $\mathrm{n}$

Relapsed

Refractory

Previous chemotherapy, $\mathrm{n}$

CHOP

$\mathrm{R}-\mathrm{CHOP}$

CHOP-E

$\geq 2$ regimens

Previous high-dose chemotherapy, $\mathrm{n}$

Previous radiotherapy, $\mathrm{n}$

NHL subtype, $\mathrm{n}$

Diffuse large B-cell lymphoma

Peripheral T-cell lymphoma
Table II. Patient responses to MEBD.

\begin{tabular}{lcccc}
\hline \multirow{2}{*}{$\begin{array}{l}\text { Patient } \\
\text { characteristic }\end{array}$} & \multicolumn{4}{c}{ Response, n (\%) } \\
\cline { 2 - 5 } All patients & $3(18.8)$ & $8(50.0)$ & $1(6.3)$ & $4(25.0)$ \\
Histological type & & & & \\
B-Cell & $2(18.2)$ & $7(63.6)$ & $0(0.0)$ & $2(18.2)$ \\
T-Cell & $1(20.0)$ & $1(20.0)$ & $1(20.0)$ & $2(40.0)$ \\
Pre-treatment status & & & & \\
Relapsed & $3(30.0)$ & $5(50.0)$ & $0(0.0)$ & $2(20.0)$ \\
Refractory & $0(0.0)$ & $3(50.0)$ & $1(16.7)$ & $2(33.3)$ \\
\hline
\end{tabular}

MEBD, mitoxantrone, etoposide, bleomycin and dexamethasone; $\mathrm{CR}$, complete response; PR, partial response; $\mathrm{SD}$, stable disease; $\mathrm{PD}$, progressive disease.

Table III. Toxicity.

Number of patients (\%)

\begin{tabular}{lllll} 
Adverse effect & Grade 1 & Grade 2 & Grade 3 & Grade 4 \\
\hline Leukopenia & $3(18.8)$ & $2(12.5)$ & $4(25.0)$ & $7(43.8)$ \\
Neutropenia & $2(12.5)$ & $3(18.8)$ & $5(31.3)$ & $6(37.5)$ \\
Anemia & $6(37.5)$ & $3(18.8)$ & $0(0.0)$ & $0(0.0)$ \\
Thrombocytopenia & $8(50.0)$ & $5(31.3)$ & $2(12.5)$ & $1(6.3)$ \\
Febrile neutropenia & $0(0.0)$ & $0(0.0)$ & $2(12.5)$ & $0(0.0)$ \\
AST/ALT elevation & $5(31.3)$ & $1(6.3)$ & $0(0.0)$ & $0(0.0)$ \\
Bilirubin elevation & $2(12.5)$ & $0(0.0)$ & $0(0.0)$ & $0(0.0)$ \\
Mucositis & $0(0.0)$ & $1(6.3)$ & $0(0.0)$ & $0(0.0)$ \\
Nausea & $6(37.5)$ & $3(13.8)$ & $0(0.0)$ & $0(0.0)$ \\
Vomiting & $4(25.0)$ & $0(0.0)$ & $0(0.0)$ & $0(0.0)$ \\
Constipation & $7(43.8)$ & $2(12.5)$ & $0(0.0)$ & $0(0.0)$ \\
Arrhythmia & $2(12.5)$ & $0(0.0)$ & $0(0.0)$ & $0(0.0)$ \\
Interstitial pneumonia & $1(6.3)$ & $3(18.8)$ & $0(0.0)$ & $0(0.0)$ \\
\hline
\end{tabular}

AST, aspartate aminotransferase; ALT, alanine aminotransferase.

ECOG, Eastern Cooperative Oncology Group; LDH, lactate dehydrogenase; CHOP, cyclophosphamide, doxorubicin, vincristine and prednisolone; CHOP-E, CHOP + etoposide; NHL, non-Hodgkin's lymphoma.

was identified in two patients (12.5\%). No renal damage or treatment-related mortality were detected.

\section{Discussion}

As the majority of patients have an incomplete or only temporary response to salvage therapy, the treatment of refractory or relapsed aggressive NHL remains a problem. Salvage chemotherapy regimens normally contain different drugs from those used previously, usually employing non-anthracycline-containing regimens to prevent drug resistance and cumulative toxicity. A number of salvage chemotherapy regimens have been developed to treat refractory or relapsed aggressive NHL. Currently, the majority of the effective salvage approaches use ifosfamide, cytarabine/platinum or gemcitabine. The most commonly used salvage regimens are dexamethasone, cytarabine and cisplatin (DHAP) (16-18), etoposide, methylprednisone, cytarabine and cisplatin (ESHAP) $(16,19)$, carmustine, etoposide, cytarabine and melphalan (mini-BEAM) $(20,21)$, ifosfamide, carboplatin and etoposide (ICE) $(18,22,23)$ and gemcitabine, dexamethasone and cisplatin (GDP) (16,24-26). These regimens have 
comparable efficacy, resulting in ORRs of $37-68 \%$ and complete remission rates of 12 to $37 \%(19,21-27)$. However, the high incidence of hematological toxicity and nephrotoxicity limits the application of these drug regimens in elderly, heavily treated or unfit patients (27).

Mitoxantrone, an anthracenedione antibiotic, exhibits similar clinical activity to the anthracyclines. Mitoxantrone intercalates into DNA through hydrogen bonding, resulting in crosslinks and strand breaks. In addition, mitoxantrone interferes with RNA and is a potent inhibitor of topoisomerase II, an enzyme responsible for uncoiling and repairing of damaged DNA $(28,29)$. In preclinical lymphoma models, the potent activity of mitoxantrone has been demonstrated, with the drug appearing to be clinically active against follicular and aggressive lymphomas (39-32). However, controversy remains with regard to the superiority of mitoxantrone or anthracyclines in the treatment of elderly NHL patients $(33,34)$. It has been hypothesized to that mitoxantrone retains the antineoplastic effects of the anthracyclines, but with less potential for cardiotoxicity, as mitoxantrone does not have the amino sugar of doxorubicin or the characteristic ring structure of the classical anthracyclines $(28,35)$. Mitoxantrone has only partial cross-resistance with anthracyclines, such as Adriamycin $(36,37)$, and the efficacy of mitoxantrone appears to be less affected by multidrug resistance than Adriamycin or etoposide $(36,38,39)$. In theory, anthracycline-resistant tumors are sensitive to mitoxantrone, and mitoxantrone may exert a synergistic effect with etoposide. The first-line use of etoposide, mitoxantrone, cyclophosphamide, vincristine, prednisolone and bleomycin (VNCOP-B) has produced an $83 \%$ ORR and a $58 \%$ CR rate in elderly patients with aggressive NHL (40). However, in this study, grade 4 neutropenia was shown to occur in $29 \%$ patients on this regimen. The combined use of etoposide, mitoxantrone and prednisone has achieved a $38 \%$ ORR among refractory or relapsed NHL patients, with relatively low toxicity (41). Therefore, in the present study, these regimens were modified and a novel combination chemotherapy regimen, MEBD, was developed, which comprises myelosuppressive (mitoxantrone and etoposide) and non-myelosuppressive (bleomycin and dexamethasone) drugs in order to increase efficacy and reduce toxicity.

In the present study, MEBD treatment, used as a salvage chemotherapeutic regimen in patients with aggressive NHL, achieved a $68.8 \%$ ORR and an $18.8 \%$ CR rate, with a median PFS of 16.7 months and a median OS of 22.4 months. Certain patients achieved long-term survival. The preliminary results appear comparable with those from patients treated with the aforementioned intensive salvage regimens, such as DHAP, ESHAP, mini-BEAM, ICE and GDP. Furthermore, the results of the present study were obtained from refractory or relapsed patients with poor health conditions, with $87.5 \%$ patients at stage III or IV. The results demonstrate that MEBD is an efficacious salvage regimen for patients with aggressive NHL who are in a state of poor health.

MEBD therapy appeared to have greater efficacy in B-cell lymphoma than in T-cell lymphoma. For the DLBCL patients, the CR rate was $18.2 \%$ and the PR rate was $63.6 \%$, thus the ORR was $81.8 \%$. Among the PTCL patients, the CR was $20.0 \%$ and the PR was $20.0 \%$, therefore the ORR was $40.0 \%$.
The refractory and relapsed NHL patients who had experienced anthracycline-based chemotherapy were observed to respond to MEBD treatment. This demonstrates that mitoxantrone-containing regimens have little cross-resistance to anthracyclines. As the present study was limited by the low number of patients, further large group studies are required in order to draw definite conclusions.

Due to the poor health conditions of the patients in the present study, hematological toxicity remained severe even if a moderate dosage of mitoxantrone and etoposide was used and the non-myelosuppressive agents bleomycin and dexamethasone were applied. Grade 3/4 neutropenia was identified in $68.8 \%$ of patients and grade $3 / 4$ thrombocytopenia was observed in $18.8 \%$ of cases. Drug doses had to be adjusted for these patients, with prophylactic G-CSF used in the following cycles of chemotherapy. Cardiotoxicity was mild even if all patients had previously received anthracycline-based chemotherapy. Only two patients $(12.5 \%)$ presented with grade 1 arrhythmia. Hepatic dysfunction was also mild; five patients $(31.3 \%)$ exhibited grade 1 and one patient $(6.3 \%)$ exhibited grade 2 transient toxicity. The gastrointestinal reactions that were detected were not severe and were controllable. Interstitial pneumonia occurred in $25.0 \%$ of patients due to the use of bleomycin. Although the interstitial pneumonia identified was classified as grade $1 / 2$ and curable, the toxicity impeded the continuation of MEBD chemotherapy. The bleomycin dose modification required to balance the treatment efficacy and the lung toxicity requires further investigation. In VNCOP-B treatment, $10 \mathrm{mg} / \mathrm{m}^{2}$ IV bleomycin was used only once every four weeks and no interstitial pneumonia was detected (40).

In conclusion, refractory or relapsed aggressive NHL patients with a poor performance status or comorbidity remain eligible for chemotherapy. MEBD is an effective and feasible salvage regimen with long-term survival efficacy for patients in a state of poor health. The most severe toxicity symptom is myelosuppression and prophylactic measures are recommended to prevent hematological toxicity.

\section{References}

1. Armitage JO and Weisenburger DD: New approach to classifying non-Hodgkin's lymphomas: clinical features of the major histologic subtypes. Non-Hodgkin's Lymphoma Classification Project. J Clin Oncol 16: 2780-2795, 1998.

2. Maxwell SA and Mousavi-Fard S: Non-Hodgkin's B-cell lymphoma: advances in molecular strategies targeting drug resistance. Exp Biol Med (Maywood) 238: 971-990, 2013.

3. Foss FM: Treatment strategies for peripheral T-cell lymphomas. Best Pract Res Clin Haematol 26: 43-56, 2013.

4. Fisher RI, Gaynor ER, Dahlberg S, et al: Comparison of a standard regimen (CHOP) with three intensive chemotherapy regimens for advanced non-Hodgkin's lymphoma. N Engl J Med 328: 1002-1006, 1993.

5. Gangatharan S and Kuruvilla J: Relapsed and refractory aggressive NHL: time for a change. Transfus Apher Sci 49: 72-79, 2013.

6. Lunning MA, Moskowitz AJ and Horwitz S: Strategies for relapsed peripheral T-cell lymphoma: the tail that wags the curve. J Clin Oncol 31: 1922-1927, 2013.

7. Peniket AJ, Ruiz de Elvira MC, Taghipour G, et al; European Bone Marrow Transplantation (EBMT) Lymphoma Registry: An EBMT registry matched study of allogeneic stem cell transplants for lymphoma: allogeneic transplantation is associated with a lower relapse rate but a higher procedure-related mortality rate than autologous transplantation. Bone Marrow Transplant 31: 667-678, 2003. 
8. Sinha R, Nastoupil L and Flowers CR: Treatment Strategies for Patients with Diffuse Large B-Cell Lymphoma: Past, Present, and Future. Blood Lymphat Cancer 2012: 87-98, 2012.

9. Mak V, Hamm J, Chhanabhai M, et al: Survival of patients with peripheral T-cell lymphoma after first relapse or progression: spectrum of disease and rare long-term survivors. J Clin Oncol 31: 1970-1976, 2013.

10. Friedberg JW: Relapsed/refractory diffuse large B-cell lymphoma Hematology Am Soc Hematol Educ Program 2011: 498-505, 2011.

11. Raut LS and Chakrabarti PP: Management of relapsed-refractory diffuse large B cell lymphoma. South Asian J Cancer 3: 66-70, 2014.

12. Oken MM, Creech RH, Tormey DC, et al: Toxicity and response criteria of the Eastern Cooperative Oncology Group. Am J Clin Oncol 5: 649-655, 1982.

13. Armitage JO: Staging Non-Hodgkin Lymphoma. CA Cancer J Clin 55: 368-376, 2005 .

14. Therasse P, Arbuck SG, Eisenhauer EA, et al: New guidelines to evaluate the response to treatment in solid tumors. European Organization for Research and Treatment of Cancer, National Cancer Institute of the United States, National Cancer Institute of Canada. J Natl Cancer Inst 92: 205-216, 2000.

15. Common Terminology Criteria for Adverse Events v3.0 (CTCAE). Cancer Therapy Evaluation Program, Common Terminology Criteria for Adverse Events, Version 3.0, DCTD, NCI, NIH, DHHS http://ctep.cancer.gov. August 9, 2006.

16. Prichard M, Harris T, Williams ME, et al: Treatment strategies for relapsed and refractory aggressive non-Hodgkin's lymphoma. Expert Opin Pharmacother 10: 983-995, 2009.

17. Velasquez WS, Cabanillas F, Salvador P, et al: Effective salvage therapy for lymphoma with cisplatin in combination with high-dose Ara-C and dexamethasone (DHAP). Blood 71: 117-122, 1988.

18. Gisselbrecht C, Glass B, Mounier N, et al: Salvage regimens with autologous transplantation for relapsed large B-cell lymphoma in the rituximab era. J Clin Oncol 28: 4184-4190, 2010.

19. Velasquez WS, McLaughlin P, Tucker S, et al: ESHAP - an effective chemotherapy regimen in refractory and relapsing lymphoma: a 4-year follow-up study. J Clin Oncol 12: 1169-1176, 1994.

20. Caballero MD, Amigo ML, Hernández JM, et al: Alternating mini-BEAM/ESHAP as salvage therapy for refractory non-Hodgkin's lymphomas. Ann Hematol 74: 79-82, 1997.

21. Girouard C, Dufresne J, Imrie K, et al: Salvage chemotherapy with mini-BEAM for relapsed or refractory non-Hodgkin's lymphoma prior to autologous bone marrow transplantation. Ann Oncol 8: 675-680, 1997.

22. Moskowitz CH, Bertino JR, Glassman JR, et al: Ifosfamide, carboplatin, and etoposide: a highly effective cytoreduction and peripheral-blood progenitor-cell mobilization regimen for transplant-eligible patients with non-Hodgkin's lymphoma. J Clin Oncol 17: 3776-3785, 1999.

23. Aurer I, Mitrović Z, Nemet D, et al: Treatment of relapsed or refractory aggressive non-hodgkin lymphoma with two ifosfamidebased regimens, IMVP and ICE. J Chemother 20: 640-644, 2008.

24. Crump M, Baetz T, Couban S, et al: Gemcitabine, dexamethasone, and cisplatin in patients with recurrent or refractory aggressive histology B-cell non-Hodgkin lymphoma: a Phase II study by the National Cancer Institute of Canada Clinical Trials Group (NCICCTG). Cancer 101: 1835-1842, 2004.

25. Crump M, Shepherd L and Lin B: A randomized phase III study of gemcitabine, dexamethasone, and cisplatin versus dexamethasone, cytarabine, and cisplatin as salvage chemotherapy followed by posttransplantation rituximab maintenance therapy versus observation for treatment of aggressive B-Cell and T-Cell non-Hodgkin's lymphoma. Clin Lymphoma 6: 56-60, 2005.
26. Kuruvilla J, Nagy T, Pintilie M, et al: Similar response rates and superior early progression-free survival with gemcitabine, dexamethasone, and cisplatin salvage therapy compared with carmustine, etoposide, cytarabine, and melphalan salvage therapy prior to autologous stem cell transplantation for recurrent or refractory Hodgkin lymphoma. Cancer 106: 353-360, 2006.

27. Hernandez-Ilizaliturri FJ and Czuczman MS: Therapeutic options in relapsed or refractory diffuse large B-cell lymphoma. Part 1. current treatment approaches. Oncology (Williston Park) 23: 546-553, 2009.

28. Wallace RE, Murdock KC, Angier RB and Durr FE: Activity of a novel anthracenedione, 1,4-dihydroxy5,8 -bis $\quad((2-[(2-$ hydroxyethyl)a mino]ethyl) amino)-9,10-anthracenedione dihydrochloride, against experimental tumors in mice. Cancer Res 39: 1570-1574, 1979.

29. Armitage JO: The role of mitoxantrone in non-Hodgkin's lymphoma. Oncology (Williston Park) 16: 490-502, 507-508, discussion 511-512, 514, 2002

30. Coltman CA Jr, Coltman TM, Balcerzak SP, et al: Mitoxantrone in refractory nonHodgkin's lymphoma. A Southwest Oncology Group study. Semin Oncol 11 (3 Suppl 1): 50-53, 1984.

31. Gams RA, Bryan S, Dukart G, et al: Mitoxantrone in malignant lymphoma. Invest New Drugs 3: 219-222, 1985

32. Silver RT, Case DC Jr, Wheeler RH, et al: Multicenter clinical trial of mitoxantrone in non-Hodgkin's lymphoma and Hodgkin's disease. J Clin Oncol 9: 754-761, 1991.

33. Chamorey E, Gressin R, Peyrade F, et al: Prospective randomized study comparing MEMID with a chop-like regimen in elderly patients with aggressive non-Hodgkin's lymphoma. Oncology 69: 19-26, 2005.

34. Mainwaring PN, Cunningham D, Gregory W, et al: Mitoxantrone is superior to doxorubicin in a multiagent weekly regimen for patients older than 60 with high-grade lymphoma: results of a BNLI randomized trial of PAdriaCEBO versus PMitCEBO. Blood 97: 2991-2997, 2001

35. Bennett JM, Muss HB, Doroshow JH, et al: A randomized multicenter trial comparing mitoxantrone, cyclophosphamide, and fluorouracil with doxorubicin, cyclophosphamide, and fluorouracil in the therapy of metastatic breast carcinoma. J Clin Oncol 6: 1611-1620, 1988.

36. Larsson R and Nygren P: Cytotoxic activity of topoisomerase II inhibitors in primary cultures of tumor cells from patients with human hematologic and solid tumors. Cancer 74: 2857-2862, 1994.

37. Klumper E,Pieters R, den Boer ML, et al: In vitro anthracycline cross-resistance pattern in childhood acute lymphoblastic leukaemia. Br J Cancer 71: 1188-1193, 1995.

38. Cole SP, Sparks KE, Fraser K, et al: Pharmacological characterization of multidrug resistant MRP-transfected human tumor cells. Cancer Res 54: 5902-5910, 1994

39. Testi R, Mattii L, Di Simone D, et al: Evaluation of resistance index of several anticancer agents on parental and resistant P-388 cell lines. Leuk Res 19: 257-261, 1995.

40. Zinzani PL, Storti S, Zaccaria A, et al: Elderly Aggressive-Histology Non-Hodgkin's Lymphoma: First-Line VNCOP-B Regimen Experience on 350 Patients. Blood 94: 33-38, 1999.

41. Doorduijn JK, Spruit P, van Der Holt B, et al: Etoposide, mitoxantrone and prednisone: a salvage regimen with low toxicity for refractory or relapsed non-Hodgkin's lymphoma. Haematologica 85: 814-819, 2000. 